\title{
Anti-CD22 Monoclonal Antibody-MMAE Conjugate DCDT2980S
}

National Cancer Institute

\section{Source}

National Cancer Institute. Anti-CD22 Monoclonal Antibody-MMAE Conjugate

DCDT2980S. NCI Thesaurus. Code C104167.

An antibody-drug conjug ate (ADC) composed of MCDT 2219A, a humanized IgG1 antiCD22 monoclonal antibody covalently linked, via a protease-cleavable peptide linker, to monomethyl auristatin $\mathrm{E}$ (MMAE), an auristatin derivative and a potent microtubule disrupting agent, with potential antineoplastic activity. Upon administration, the monoclonal antibody moiety of DCDT2980S binds to B cell-specific CD22 receptors and is rapidly internalized, thereby delivering MMAE intracellularly. Upon proteolytic cleavage, MMAE binds to tubulin and inhibits its polymerization, resulting in G2/M phase arrest and tumor cell apoptosis. CD22, a cell surface glycoprotein, is expressed on mature B-cells and on most malignant B-cells. 